\title{
UCRL-JRNL-216651
}

LAW RENCE LIVERMORE N A TIO NAL LABORATORY

\section{Transport and Fluctuations in High Temperature Spheromak Plasmas}

H. S. McLean, S. Woodruff, R. D. Wood, E. B. Hooper, D. N. Hill, J. Moller, C. Romero-Talamas

October 28, 2005

Physics of Plasmas 
This document was prepared as an account of work sponsored by an agency of the United States Government. Neither the United States Government nor the University of California nor any of their employees, makes any warranty, express or implied, or assumes any legal liability or responsibility for the accuracy, completeness, or usefulness of any information, apparatus, product, or process disclosed, or represents that its use would not infringe privately owned rights. Reference herein to any specific commercial product, process, or service by trade name, trademark, manufacturer, or otherwise, does not necessarily constitute or imply its endorsement, recommendation, or favoring by the United States Government or the University of California. The views and opinions of authors expressed herein do not necessarily state or reflect those of the United States Government or the University of California, and shall not be used for advertising or product endorsement purposes. 


\section{Transport and fluctuations in high temperature spheromak plasmas.}

H.S. McLean, S. Woodruff, R.D. Wood, E.B. Hooper, D.N. Hill, J. Moller, Carlos RomeroTalamas

Lawrence Livermore National Laboratory

\section{Abstract}

Globally coherent magnetic fluctuations often observed during the driven phase after spheromak formation in the Sustained Spheromak Physics Experiment ${ }^{1}$ (SSPX) can be reduced to small amplitude by programming the magnetic flux $=\psi_{\text {gun }}$ and the discharge current $=I_{\text {gun }}$ in the formation gun. Scanning the edge normalized current $=\lambda_{\text {edge }}=\lambda_{\text {gun }}=\mu_{0} I_{\text {gun }} / \psi_{\text {gun }}$ above and below the minimum energy eigenvalue ${ }^{2}=\lambda_{\mathrm{FC}}$ of the flux conserver provides a variation in the internal $\mathrm{q}=$ safety factor profile producing the expected $\mathrm{q}=\mathrm{m} / \mathrm{n}=$ poloidal/toroidal mode spectrum. $\mathrm{By}$ driving the edge with the proper $\lambda_{\text {gun }}$, the system can be operated with the poloidal/toroidal mode spectrum between the $\mathrm{m} / \mathrm{n}=1 / 2$ and $2 / 3$ modes producing low magnetic fluctuation amplitudes and high electron temperature $=\mathrm{T}_{\mathrm{e}}>350 \mathrm{eV}$. Transport and confinement parameters calculated using Thomson scattering-measured $\mathrm{T}_{\mathrm{e}}$ and $\mathrm{n}_{\mathrm{e}}$ profiles coupled with the equilibrium code internal current profiles show a reduction in electron thermal diffusivity as $T_{e}$ increases. This scaling behavior is more classical-like than Bohm or open field line transport models ${ }^{3}$ where thermal diffusivity increases with $T_{\mathrm{e}}$. Electron diffusivity is calculated to be less than $10 \mathrm{~m}^{2} / \mathrm{s}$, approaching levels seen in tokamaks.

\section{INTRODUCTION}

Spheromaks ${ }^{4}$ formed with a single pulse followed by free decay in a flux conserver with a magnetized gun initially relax to a stable, minimum energy Taylor-State ${ }^{2}$ with a well defined ratio of poloidal to toroidal current, but then suffer from internal kink (or resistive tearing) mode instability $^{5,6,7,8}$ [Janos, Knox, Sgro, Ono] as the colder, more resistive edge causes the poloidal current to decay faster than the toroidal current causing a loss of toroidal magnetic field. This instability is often disruptive enough to rapidly terminate the plasma discharge and is consistent 
with the internal normalized current profile $\lambda=\mu_{0} \mathrm{j} / \mathrm{B}$ and safety factor profile $\mathrm{q}=\mathrm{d} \psi / \mathrm{d} \phi$ with $\psi=$ toroidal flux, $\phi=$ poloidal flux. Flatter $\lambda$ profiles are more stable; profiles peaked (or hollow) at the magnetic axis can cause the q-profile to span mode-resonant surfaces. In the free decay case, the loss of toroidal magnetic field causes a peaking in the $\lambda$ profile at the magnetic axis leading $\mathrm{q}$ to span the $m / n=1 / 2$ surface.

During the formation pulse, the gun current flowing down the open field lines near the geometric axis and returning near the outer wall back to the gun is very large with the q-profile hollow and greater than 1 , much like a tokamak. With this large drive on the edge, the $\mathrm{q}=\mathrm{m} / \mathrm{n}=1 / 1$ dough-hook ${ }^{9,10,11}$ mode is active (generating toroidal current and forming the spheromak configuration) but the fluctuation amplitudes are large and open field lines limit plasma temperature $^{12}$ [Moses]. After the formation pulse, gun current drops, and q drops below one to a reverse-shear profile: $\mathrm{q}$ is peaked at the magnetic axis, a minimum near the outer edge, and diverges at the separatrix. Flux surfaces form, confinement improves and ohmic heating raises Te. Without continued edge drive, resistive dissipation in the colder edge region causes $\lambda$ to sag at the edge and peak at the magnetic axis lowering $q$ at the edge to $1 / 2$ and then $1 / 3$. The plasma responds by relaxing towards minimum energy, converting toroidal current into poloidal current ${ }^{13}$ [Ono] through the $\mathrm{q}=\mathrm{m} / \mathrm{n}=1 / 2$ and $1 / 3$ modes now driven by the peaked $\lambda$ profile. Often, the $\mathrm{m} / \mathrm{n}=1 / 2$ mode is sufficient to cause disruptive termination of the configuration before higherorder $n=3,4$ modes are observed and before relaxation to minimum energy is realized.

It is shown here that driving edge current for an extended period of time after formation prevents decay of the poloidal current, suppresses the $m / n=1 / 2$ mode, and allows the system to decay on the timescale of the toroidal current flowing in the hotter and more conductive core thus greatly increasing the plasma lifetime. Most significantly, the q profile can be controlled during the discharge by adjusting the initial flux configuration and programming the discharge current. It is possible in SSPX to maintain the q-profile between resonant surfaces, precluding large global 
fluctuation for several milliseconds (up to the charge capacity of the sustainment capacitor bank), a much longer duration than previously ${ }^{1}$ achieved. We have identified that operating between the $2 / 3$ and $1 / 2$ modes produces the highest $\mathrm{Te}(>350 \mathrm{eV})$ yet observed in a spheromak driven for these longer periods of time. On a smaller-volume (18 \% of SSPX), higher magnetic field (600\% of SSPX) configuration of $\mathrm{CTX}^{14}$, several shots with $\mathrm{Te}>200 \mathrm{eV}$ (one with $\mathrm{Te} \sim 400 \mathrm{eV}$ ) were measured near peak magnetic field. These discharges had a formation pulse width of $200 \mathrm{usec}$, magnetic field peaking at $\sim 300$ usec decaying to $1 / 2$ maximum at $\sim 600$ usec.

\section{Results and Discussion}

\section{A. Description of SSPX geometry and operation}

SSPX geometry is show in Fig. 1. Typical discharge parameters are shown in Table 1 and time histories of several parameters are shown in Figure 2. The time sequence for forming and sustaining a spheromak on SSPX is as follows: The external magnetic field bias coils are energized several seconds before a discharge to allow the bias magnetic flux to diffuse through the thick $(20 \mathrm{~mm})$ copper wall of the flux conserver. Fast-puff gas valves are fired $\sim 250$ usec before discharge to allow gas to flow into the region between the electrodes. A formation capacitor bank $(10 \mathrm{mF}, 10 \mathrm{kV})$ is discharged which ionizes the gas (hydrogen) and produces a large initial pulse of $500 \mathrm{kA}$ (Fig 2a). A second, sustaining capacitor bank is then fired. This bank $(120 \mathrm{mF}, 5 \mathrm{kV})$ is arranged as a 5 -stage pulse-forming network to give a relatively constant flattop current after formation. The large initial current pulse forms the spheromak as shown by the buildup of magnetic field to about $.34 \mathrm{~T}$ at the outer midplane edge (Fig. 2b.) Gun current then drops and the flat-top current maintains the spheromak with edge magnetic fluctuations that evolve over time as shown in Fig 2c. The plasma density (Fig. 2d) is high during formation, but falls to a constant level of $10^{20} \mathrm{~m}^{-3}$ (chord-averaged) until the end of the shot. Finally, the electron 
temperature is observed to increase to a peak value of $\sim 350 \mathrm{eV}$ and then decay till the end of the shot.

\section{B. Optimum $\lambda_{\text {edge }}$ produces highest Te.}

A series of shots were taken varying the gun flux from $20-30 \mathrm{mWb}$ while holding the sustainment bank charge voltage constant (thus varying $\lambda_{\text {edge }}$ ) to determine the effect. Sustainment bank current varied slightly at constant charge voltage when changing the flux but was nominally

$230 \mathrm{kA}$. This produced a variation in $\lambda_{\text {edge }}\left(6 \mathrm{~m}^{-1}<\lambda_{\text {edge }}<12 \mathrm{~m}^{-1}\right)$ above and below the eigenvalue of the flux conserver, $\lambda_{\mathrm{fc}}=9.5 \mathrm{~m}^{-1}$. Thomson scattering measurements of $\mathrm{T}_{\mathrm{e}}$ were taken $2 \mathrm{~ms}$ after breakdown, the expected peak in $T_{e}$. The results, plotted as blue x's in Fig. 3, clearly show an optimum $\lambda_{\text {edge }}$ at about $9 \mathrm{~m}^{-1}$, slightly less than $\lambda_{\mathrm{fc}}$. Later, the sustainment bank charge voltage was raised to its rated maximum voltage and a few shots were taken. This produced a sustainment bank current of $260 \mathrm{kA}$. The flux was also raised to maintain $\lambda_{\text {edge }}$ in the optimum range. These shots, shown as red circles in Fig. 3 resulted in the highest $T_{e}$ yet observed on SSPX and close to the $400 \mathrm{eV}$ observed on CTX. Radial profiles of six high $\mathrm{T}_{\mathrm{e}}$ shots are plotted in Fig.4.

\section{Evolution of magnetic fluctuations during a discharge}

Signals from an array of 14 Rogowski coils on the mid-plane posts are Fourier analyzed to determine the instantaneous amplitude and phase of toroidal spatial variations in the wall return current. The signals are decomposed into modes up to $n=5$. The analysis looks at signal frequencies of 1-100 kHz. The typical evolution of modes is shown in Fig. 5 along with the gun current and electron temperature measurements. A large amplitude $n=1$ is observed during the time the field is initially building in agreement with observations on the SPHEX experiment ${ }^{11}$ and NIMROD modeling ${ }^{15}$. After the formation current drops, an $n=2$ is observed followed by a relatively mode-free period where the electron temperature reaches its highest value. Later, an $\mathrm{n}=3$ often develops. 


\section{Equilibrium reconstructions show optimal $\lambda_{\text {edge }}$ produces $1 / 2<q<2 / 3$.}

The mode evolution can be understood by considering the internal safety factor q profile from equilibrium reconstructions. For rational values of q, global magnetic fluctuations are expected with mode numbers $\mathrm{q}=\mathrm{m} / \mathrm{n}=$ poloidal/toroidal mode numbers. The CORSICA code [Hooper, et al] reconstructs the internal field and currents by fitting to edge magnetic probes, gun current, initial magnetic flux, and a parameterization of $\lambda$ given by $\lambda=\lambda_{0}\left(1+\alpha_{4} \bar{\psi}^{2}\right) ; \lambda_{0}$ is the value on the magnetic axis, $\bar{\psi}=\left(\psi-\psi_{0}\right) /\left(\psi_{\text {edge }}-\psi_{0}\right)$ is the normalized poloidal flux with $\psi_{0}$ the value at the magnetic axis. The fitting parameter, $\alpha_{4}$, is positive for hollow profiles and negative when peaked.

A reconstruction of poloidal flux contours for optimum $\lambda_{\text {edge }}$ during the mode-free period is shown in Fig. 6 with a corresponding plot of $\lambda$, q, and Te spatial profiles shown in Fig. 7. The equilibrium shows $1 / 2<\mathrm{q}<2 / 3$ and a peaked $\lambda$ profile in agreement with the observations that mode amplitudes are low during this interval. Fig. 7 shows that the peak in the $T_{e}$ profile is aligned with the magnetic axis. Fig. 8 overlays $T_{e}$ vs. $\psi$ for $T_{e}$ measured at major radius $R>$ $\mathrm{R}_{\text {magnetic axis }}$ with $\mathrm{T}_{\mathrm{e}}$ vs. $\psi$ for $\mathrm{T}_{\mathrm{e}}$ measured at $\mathrm{R}<\mathrm{R}_{\text {magnetic axis. These should overlay if the }}$ reconstructed flux surfaces are isothermal, which indeed they do. This is good independent confirmation of the validity of the flux surface reconstruction.

\section{E. Global fluctuations observed when $\lambda_{\text {edge }}$ not optimum.}

As $\lambda_{\text {edge }}$ is varied from optimum, the q-profile is varied and the expected magnetic fluctuating global modes are observed. Figure 9a shows a plot of safety factor $\mathrm{q}$ at the magnetic axis, $\mathrm{q}_{\mathrm{o}}$ and the minimum in $\mathrm{q}$ near the separatrix, $\mathrm{q}_{\min }$, during a discharge along with amplitudes of the $n=2$ and $n=3$ toroidal modes. $\lambda_{\text {edge }}$ varies with the discharge current producing the change in $\mathrm{q}$. When $\mathrm{q}_{\min }$ drops below $1 / 2$, the amplitude of the $\mathrm{n}=2$ mode increases and then drops out when $\mathrm{q}_{\min }$ rises above $1 / 2$. The mode appears again near the end of the shot when the $\mathrm{q}=1 / 2$ 
surface passes through the plasma again. Figure $9 \mathrm{~b}$ shows a discharge where the $\mathrm{q}$ went too high and excited the $n=2 / 3$ mode.

CORSICA equilibrium reconstructions provide the relationship between $\mathrm{q}$ and $\lambda$ for SSPX. This is plotted in Fig. 10a. The $\mathrm{q}=1 / 2$ and $\mathrm{q}=2 / 3$ stability boundaries are identified and overlaid on the multi-shot temperature data in Figure $10 \mathrm{~b}$. Te is consistently higher in the modefree region and indicates that global magnetic fluctuations are a likely candidate responsible for low Te outside the optimum range of $\lambda_{\text {edge. }}$ The mapping of these boundaries provides valuable guidance in operating the experiment.

An upgrade is in progress to double the sustainment bank energy and implement flexible, pulse-shaping solid-state switching. This will allow for longer plasma discharges and more options for controlling the sustainment current during the discharge. This will extend the modefree interval from $2.5 \mathrm{~ms}$ out to $8 \mathrm{~ms}$.

\section{F. Transport profiles show good confinement in the core.}

Transport profiles show thermal diffusivity $\chi_{\mathrm{e}}<10 \mathrm{~m}^{2} / \mathrm{s}$ in the core of SSPX, approaching the values measured in tokamaks. Profiles are calculated by the CORSICA code by setting heat flow out of each flux surface to the internal heating within each flux surface. Te and ne profiles are obtained by fitting Thomson-scattering measurements onto equilibrium reconstructed flux surfaces. The calculation assumes steady state, ohmic heating only (ignores ion heating and transport), radiation power $=0, \mathrm{~T}_{\mathrm{e}}$ dependent Spitzer resistivity, and Zeff $=2.3$. Plots of $\mathrm{T}_{\mathrm{e}}$, ohmic heating density, and $\chi_{\mathrm{e}}$ are shown in Figure11. The difference between the $\mathrm{T}_{\mathrm{e}}$ profiles for the various shots is representative of the shot-to-shot variation in $T_{e}$. Ohmic power is highest at the edge where $T_{e}$ is lower and resistivity higher. For $r / a<0.4$ however, the ohmic dissipation maintaining the high Te is very low indicating a good confinement region. How this core to edge relationship scales with size and perhaps other parameters will be important in determining the practicality of an eventual spheromak power reactor. 


\section{G. $\chi_{e}$ in core near tokamak levels, scales as $\mathrm{T}_{\mathrm{e}}^{-5 / 2}$}

Scaling $\chi \mathrm{e}$ vs. Te shows the regimes where various transport models may apply. Below $100 \mathrm{eV}$, stochastic behavior may apply. This line is indicated by RR for Rechester-Rosenbluth. Thermal diffusivity in this regime can be given by ${ }^{16}: \chi_{R R}=v_{t h} L_{c}(d B / B)^{2}$ where $v_{\text {th }}$ is the electron thermal velocity, $\mathrm{L}_{\mathrm{c}}$ is a correlation length taken to be the short of a collision length or one toroidal transit distance, and $\mathrm{dB} / \mathrm{B}$ is the fluctuation amplitude in the radial direction. Magnetic fluctuations can be large in this temperature range driven by the previously described global fluctuations. Moving to higher $T_{e}$, Bohm diffusion given by $D_{B o h m}=T_{e} / 16 B$ fits the data. At the highest Te however, the thermal diffusivity is approaching the ion classical diffusivity given by $\chi_{\mathrm{i}}$ $=\rho_{\mathrm{i}}{ }^{2} / \tau_{\mathrm{i}}$ where $\rho_{\mathrm{i}}$ is the ion Larmor radius and $\tau_{\mathrm{i}}$ is the ion collision time. This low value of calculated $\chi_{\mathrm{e}}$ at the highest Te indicates that it is important to include effects not included in the present analysis particularly the contribution due to the ions. Hot ions have been observed consistently in spheromaks during formation and reconnection. Ion heating may play a large role in the initial heat-up of the spheromak. Additional work is planned to include the ion channel. The upgrade to the sustaining current system to extend the discharge time will allow more time for transients to die improving the calculation of transport properties.

\section{Summary}

Globally coherent magnetic fluctuations are observed during the driven phase after spheromak formation in the Sustained Spheromak Physics Experiment (SSPX) by analysis of edge magnetic probe signals. The observed modes are low order $\mathrm{m} / \mathrm{n}=\mathrm{q}$ ( safety factor)=poloidal/toroidal=1/2,2/3 and correlate with the internal q-profiles inferred by equilibrium reconstructions using the CORSICA $^{17}$ code. The fluctuation mode numbers and amplitudes can be controlled to a large extent by programming the magnetic flux and the discharge current Scanning the $\lambda_{\text {edge }}$ above and below the minimum energy eigenvalue $=\lambda_{\mathrm{FC}}$ of the flux conserver provides a variation in the 
internal q profile producing the expected mode spectrum. By driving the edge with the proper $\lambda_{\text {edge, }}$ the system can be operated with the mode spectrum between the $1 / 2$ and $2 / 3$ modes producing low magnetic fluctuation amplitudes and high electron temperature $=\mathrm{T}_{\mathrm{e}}>350 \mathrm{eV}$. Since CORSICA does a good job predicting the mode spectrum, there is some confidence in the internal current profile generated by the code. Transport and confinement parameters calculated using Thomson scattering-measured $\mathrm{T}_{\mathrm{e}}$ and $\mathrm{n}_{\mathrm{e}}$ profiles coupled with the CORSICA current profile shows a reduction in electron thermal diffusivity as Te increases. This scaling behavior is more classical-like than Bohm or open field line transport models where thermal diffusivity increases with $\mathrm{T}_{\mathrm{e}}$.

\section{Acknowledgements}

This work was performed under the auspices of US DOE by the University of California Lawrence Livermore National Laboratory under Contract No. W-7405-ENG-48. 


\section{References}

${ }^{1}$ H.S. McLean, S. Woodruff, E.B. Hooper, R.H. Bulmer, D.N. Hill, C. Holcomb, J. Moller, B.W. Stallard, R.D. Wood, Z. Wang, "Suppression of MHD Fluctuations Leading to Improved Confinement in a Gun-Driven Spheromak," Phys. Rev. Lett. 88, 125004 (2002).

2 J.B. Taylor, Phys. Rev. Lett. 33, 1139 (1974).

${ }^{3}$ R. Moses, R.A. Gerwin, K.F. Shoenberg, "Transport implications of current drive by magnetic helicity injection," Phys. Plasmas, 8, 4839 (2001).

${ }^{4}$ M.N. Rosenbluth and M.N. Bussac, Nucl. Fusion 19, 489 (1979).

${ }^{5}$ A. Janos, G.W. Hart, C.H. Nam, M. Yamada, "Global magnetic fluctuations in spheromak plasmas and relaxation toward a minimum-energy state,” Phys. Fluids 28, 3667 (1985).

${ }^{6}$ S.O. Knox, C.W. Barnes, G.J. Marklin, T.R. Jarboe, I. Henins, H.W. Hoida, B.L. Wright, "Observations of Spheromak Equilibria Which Differ from the Minimum Energy State and Have Internal Kink Distortions,” Phys. Rev. Lett. 56, 842 (1986).

${ }^{7}$ A.G. Sgro, A.A. Mirin, G. Marklin, “The evolution of a decaying spheromak,” Phys.Fluids 30, 3219 (1987).

${ }^{8}$ Y. Ono, R.A. Ellis, A.C. Janos, et. al., "Relaxation Phenomena in the High-Temperature S-1 Spheromak,” Phys. Rev. Lett. 61, 2847 (1988).

${ }^{9}$ L. Lindberg, C. Jacobson, "On the amplification of the poloidal magnetic flux in a plasma," Astrophys. J., 133, 1043 (1961)

${ }^{10}$ R. Duck, et al., PPCF 39, 715 (1997).

${ }^{11}$ P.K. Browning, G. Cunningham, S.J. Gee, et. al., "Power Flow in a Gun-Injected Spheromak," Phys. Rev. Lett. 68, 1718 (1992).

${ }^{12}$ R. Moses, R.A. Gerwin, K.F. Shoenberg, “Transport implications of current drive by magnetic helicity injection," Phys. Plasmas, 8, 4839 (2001).

${ }^{13}$ Y. Ono, M. Yamada, A.C. Janos, F.M. Levinton, "Experimental study of the relaxation cycle of a decaying spheromak in an external magnetic field," Phys. Fluids B 3, 1452 (1991).

${ }^{14}$ T. Jarboe, et. al., Phys Fluids B, 2, 1346 (1990).

${ }^{15}$ B. Cohen, et. al., Physics of Plasmas 12, 056156 (2005).

${ }^{16}$ A.B. Rechester and M.N. Rosenbluth, Phys. Rev. Lett. 40, 38 (1978).

${ }^{17}$ E.B.Hooper, L.D. Pearlstein, and R.H. Bulmer, Nucl. Fusion 39, 863 (1999). 
Figures

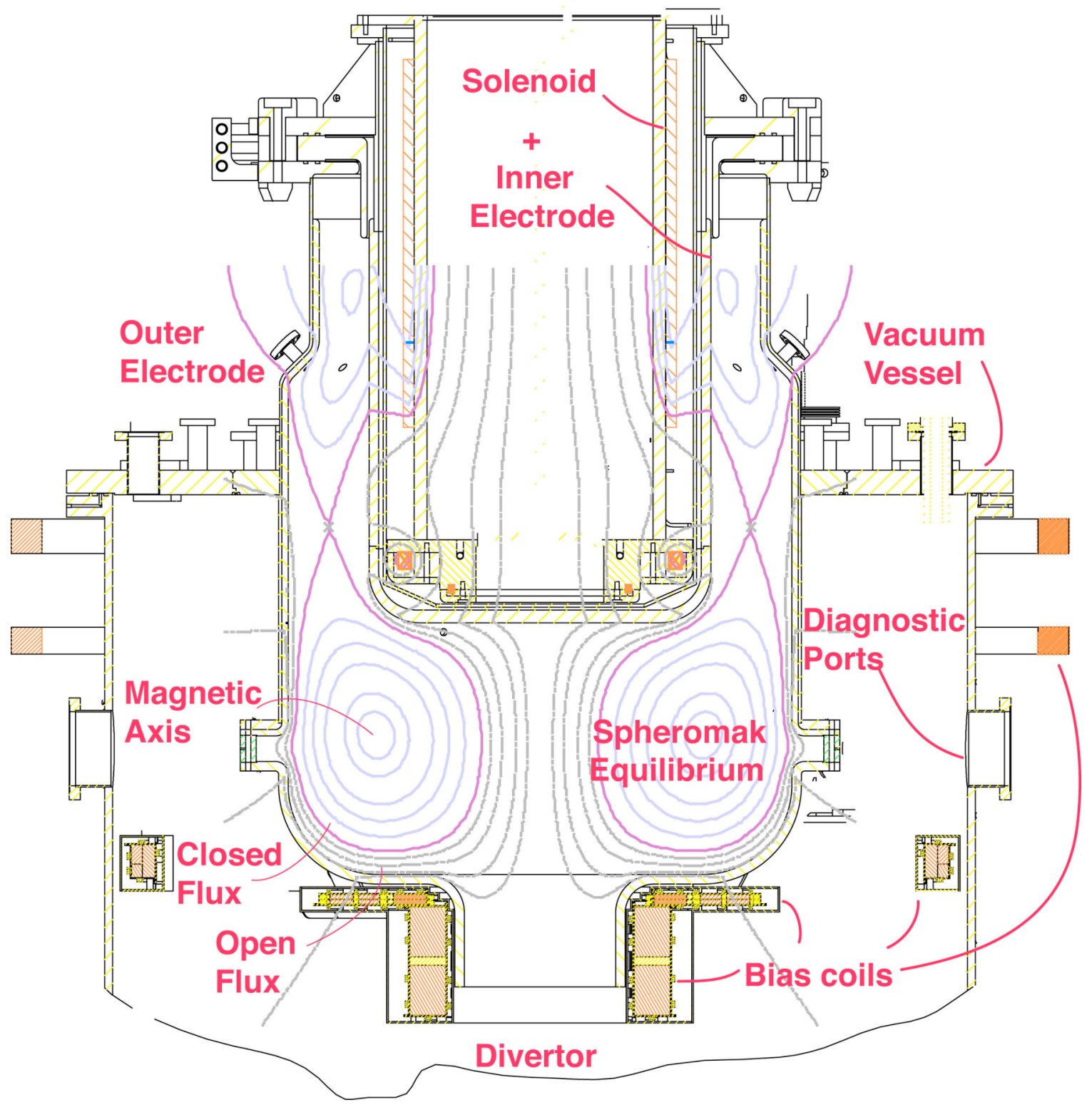

Figure 1. (Color) Side view of SSPX. 


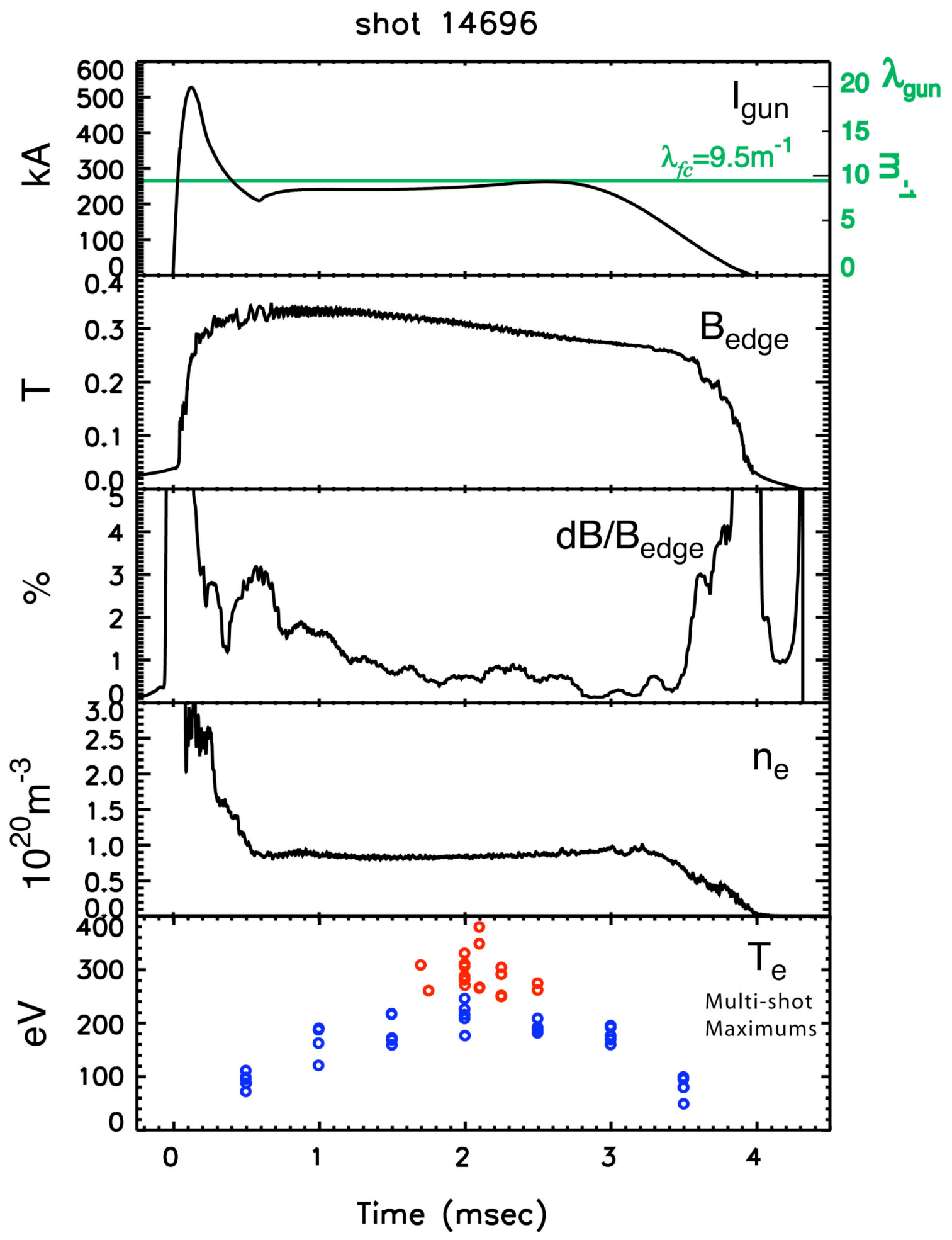

Figure. 2 (color) Discharge data for $\mathrm{Te}=230 \mathrm{eV}$ shot. 


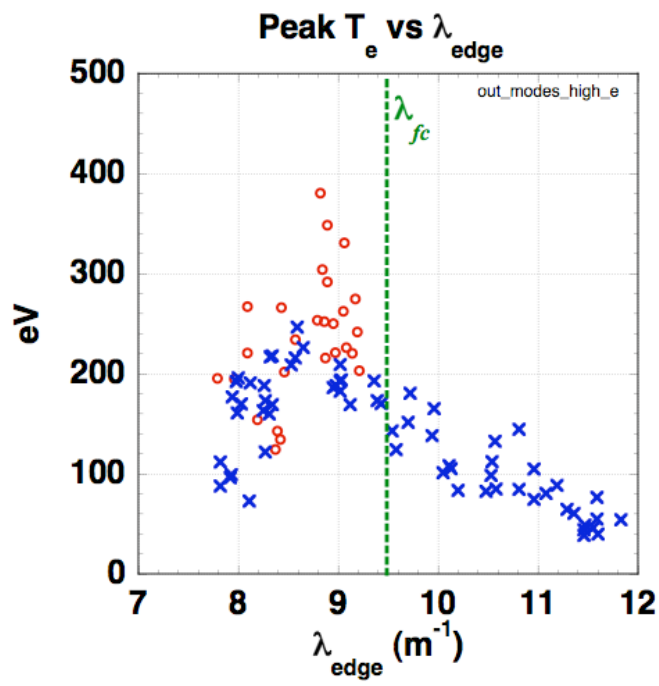

Figure 3. Highest $\mathrm{T}_{\mathrm{e}}$ is observed with $\lambda_{\text {edge }}<\lambda_{\mathrm{fc}}$

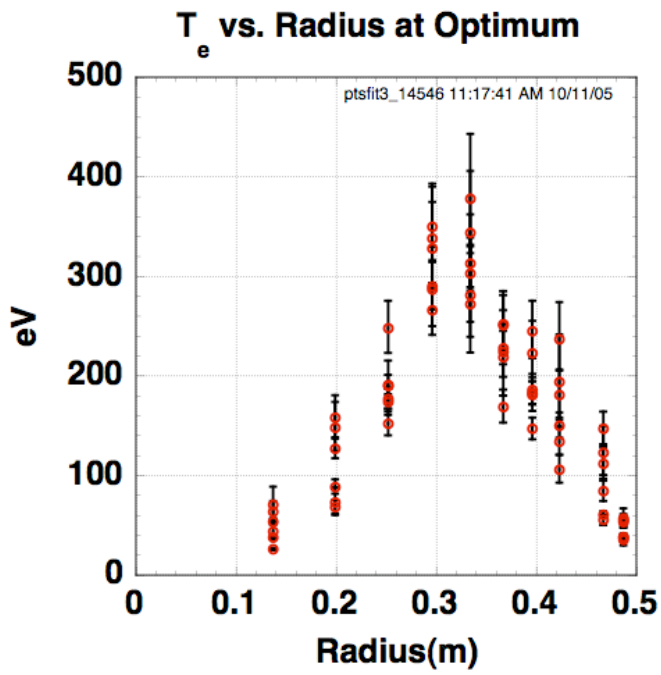

Figure $4 . T_{e}$ profile at maximum available sustainment bank current and optimum $\lambda_{\text {edge }}$. 


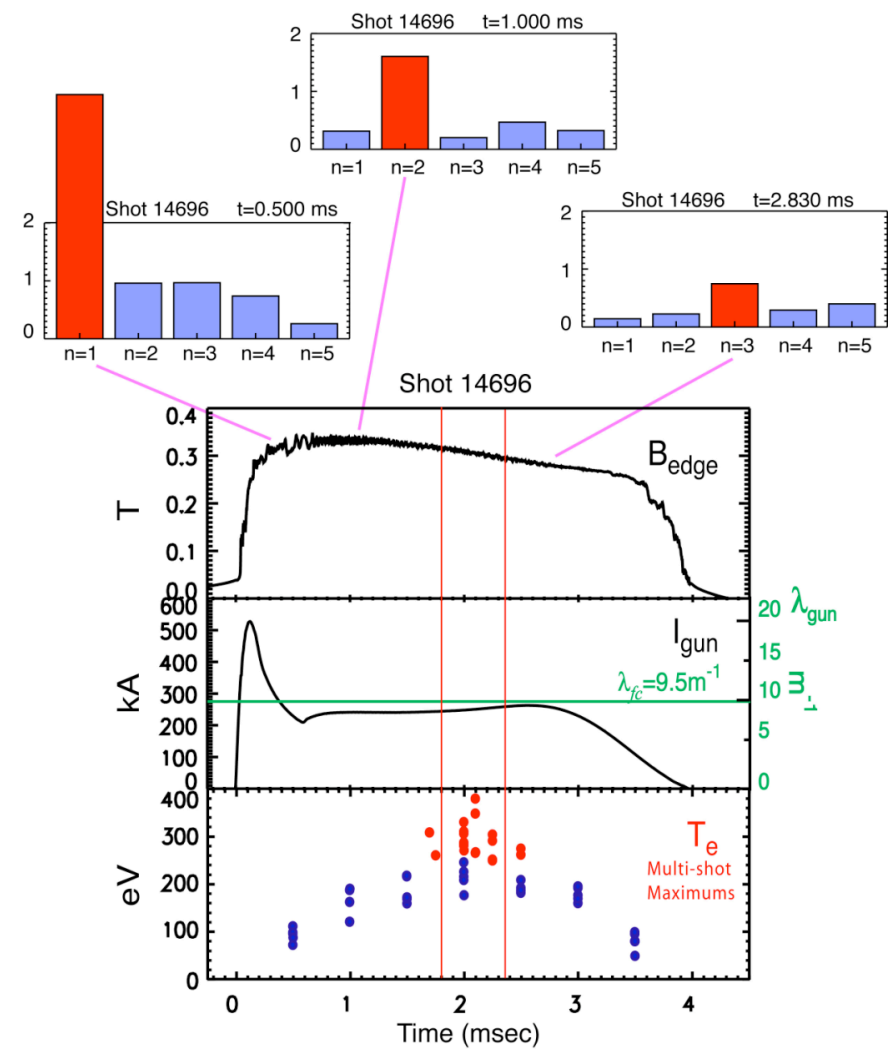

Figure 5. Modes evolve from $n=1$ during formation to $n=2$ to a mode-free period to an $n=3$.

Highest temperatures are observed during the mode-free period between the $n=2$ and $n=3$. 


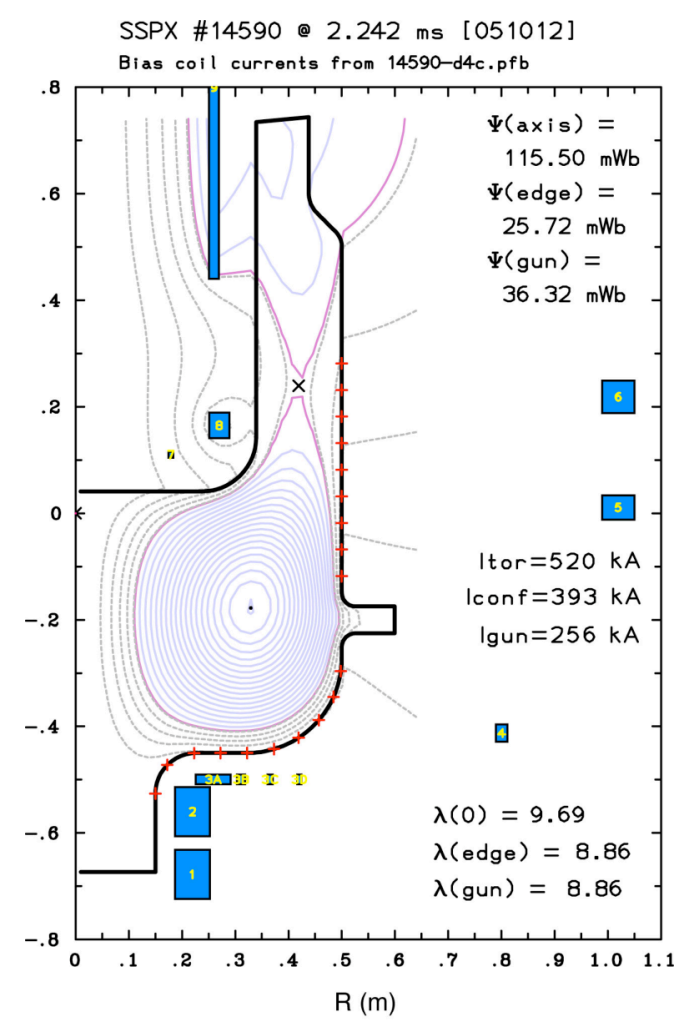

Fig 6. Internal profiles from CORSICA equilibrium fitting shows flux contours and reconstruction parameters. 


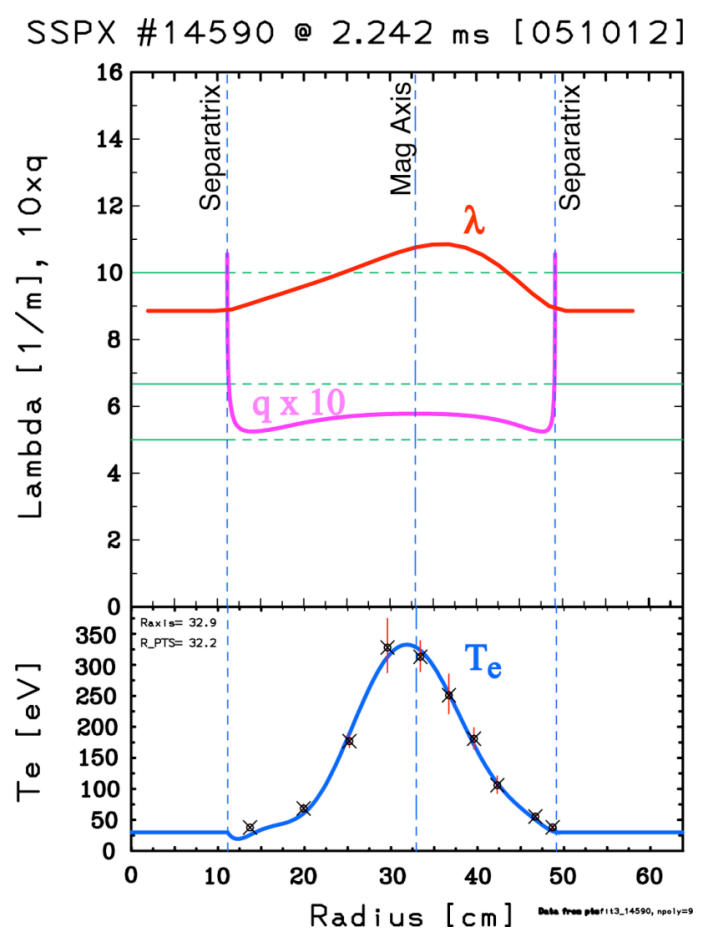

Fig 7 . Internal $\lambda$ and q profile from equilibrium. Te profile is peaked at the magnetic axis. 


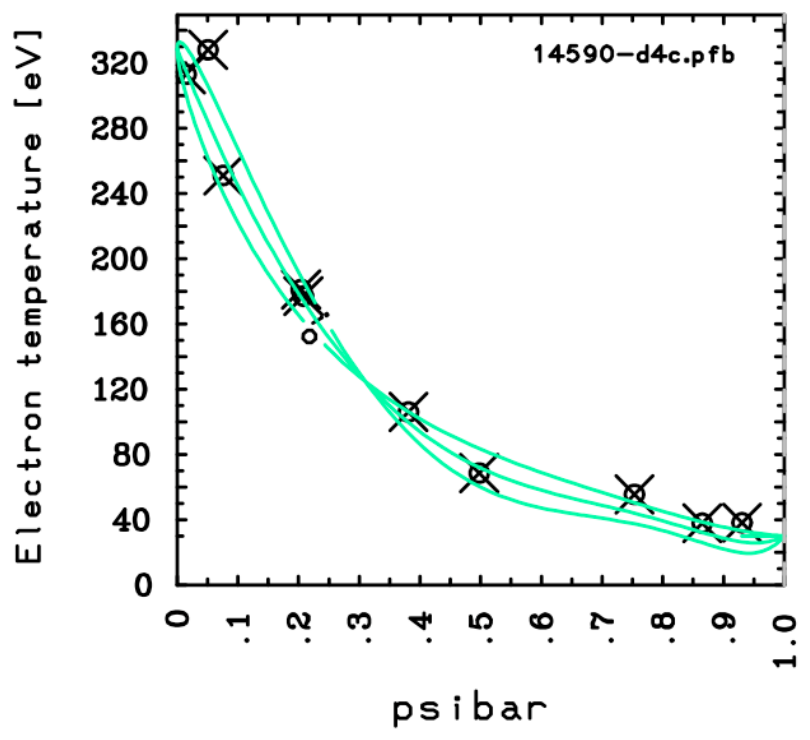

Fig. 8. Te aligns with reconstructed flux surfaces inboard and outboard of the magnetic axis. 

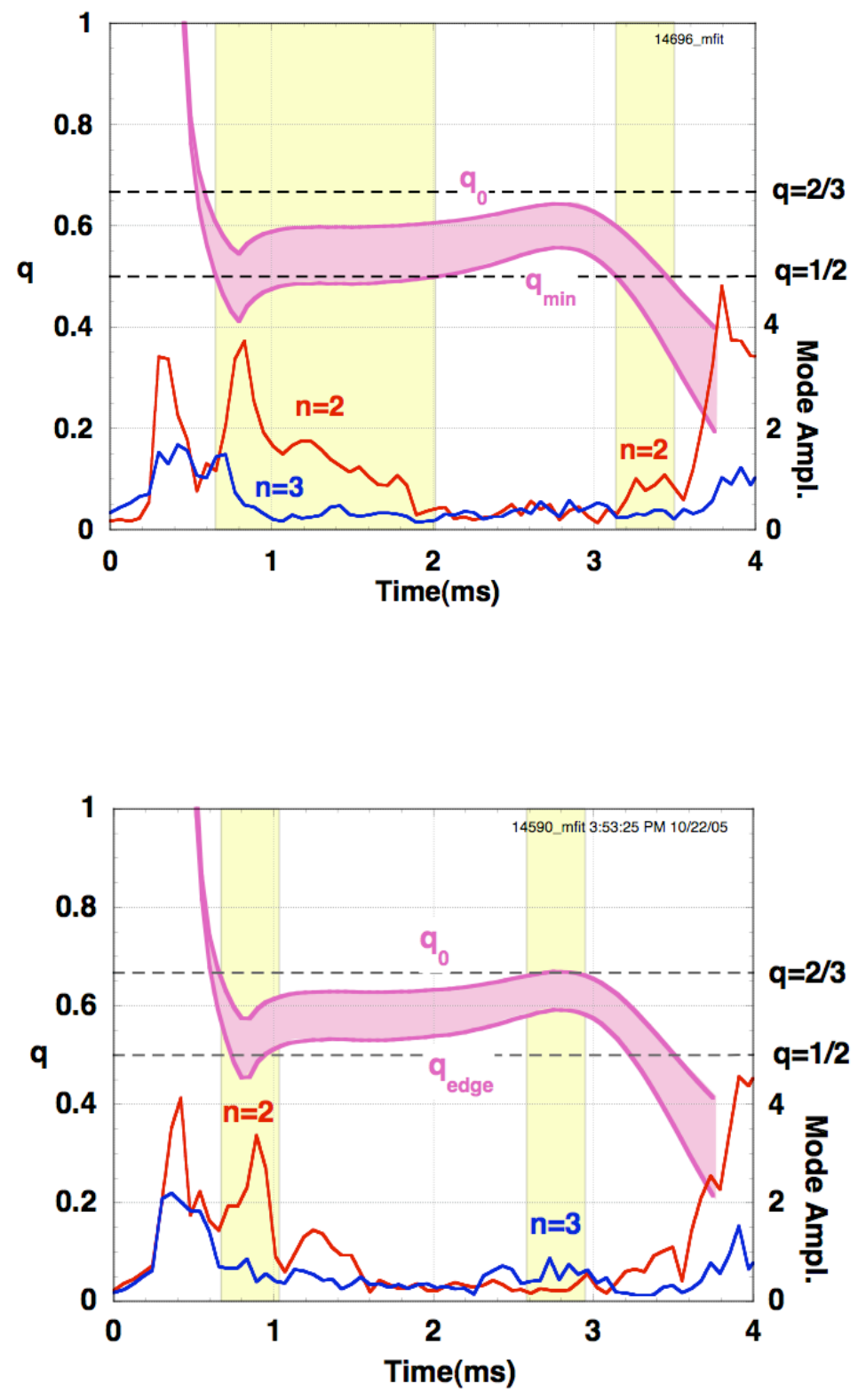

Figure 9 The expected modes are observed as the safety factor q spans different rational surfaces during the discharge. a) The $n=2$ mode is excited at the beginning and end of the shot. b) $q$ is higher for this shot reducing the duration of $n=2$, but getting high enough at the end to excite the $n=3$ mode. There is a narrow range of mode-free operation. 

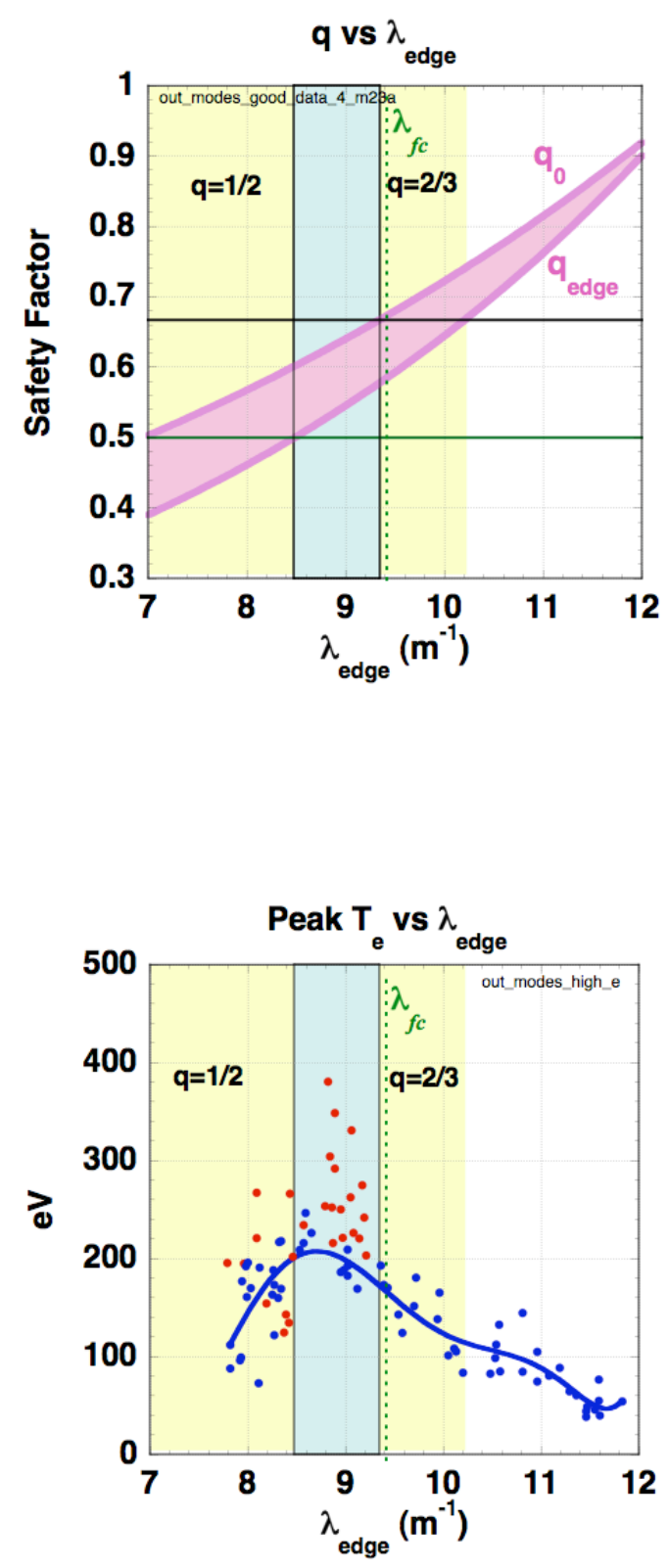

Figure 10. a) Safety factor vs. ledge showing stability boundaries for $m / n=1 / 2,2 / 3$ modes. b) Overlay of stability boundaries on the Te data of Figure 3 showing high Te between the unstable regions. 

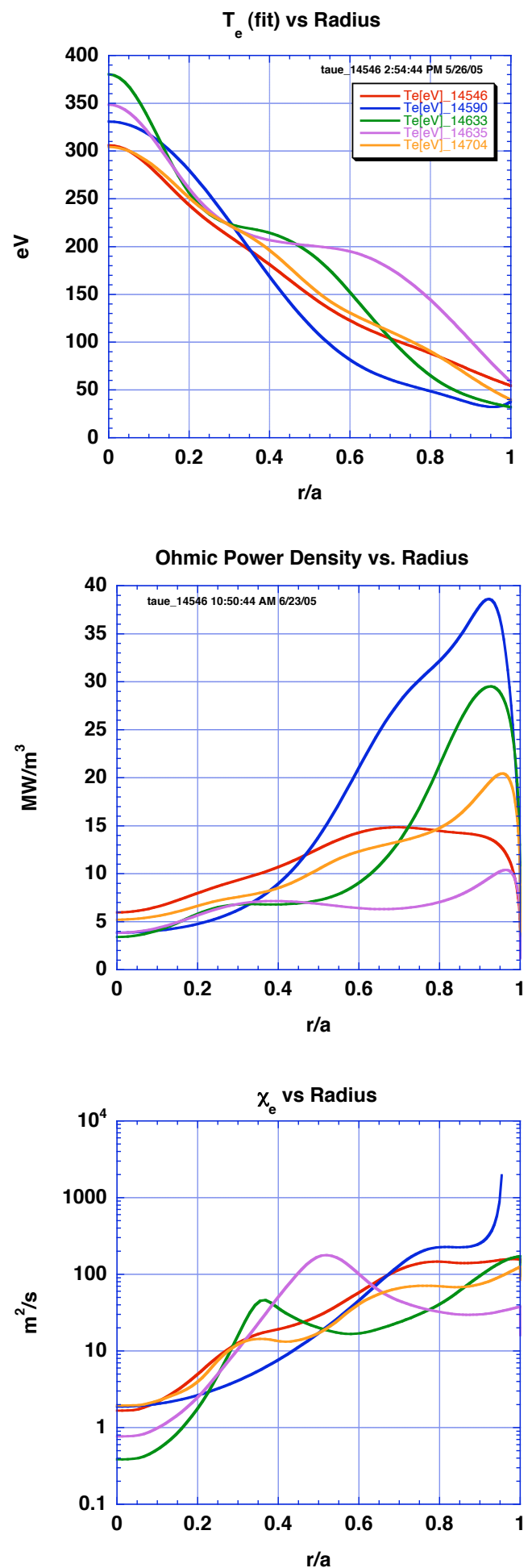

Figure 11. Transport properties of SSPX $350 \mathrm{eV}$ shots. 


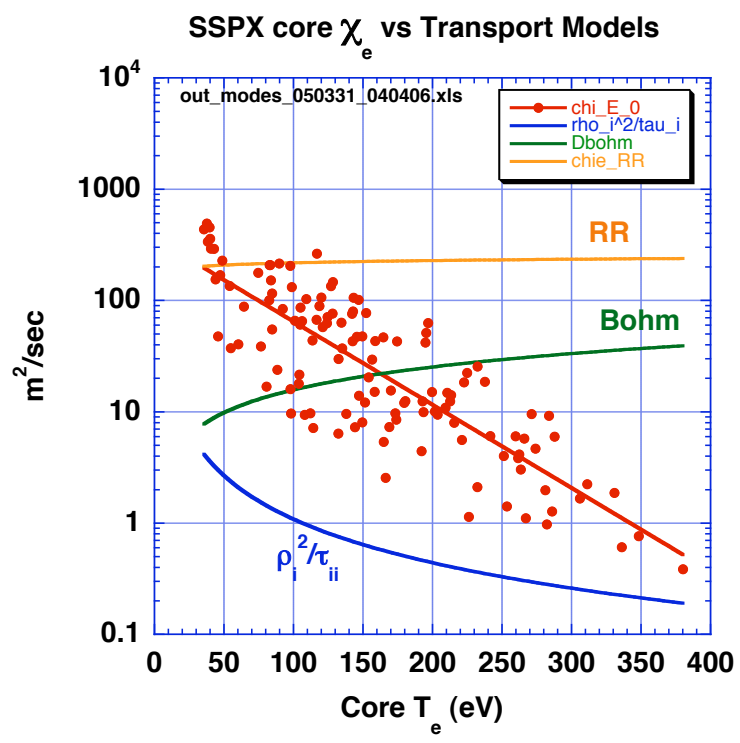

Figure 12. Scaling of $\chi_{\mathrm{e}}$ with Te along with various transport models. 